\title{
MRI Atlas-Based Measurement of Spinal Cord Injury Predicts Outcome in Acute Flaccid Myelitis
}

\author{
(DD.B. McCoy, (D).F. Talbott, (D)Michael Wilson, (DM.D. Mamlouk, (D). Cohen-Adad, (DMark Wilson, and (D). Narvid
}

\begin{abstract}
BACKGROUND AND PURPOSE: Recent advances in spinal cord imaging analysis have led to the development of a robust anatomic template and atlas incorporated into an open-source platform referred to as the Spinal Cord Toolbox. Using the Spinal Cord Toolbox, we sought to correlate measures of GM, WM, and cross-sectional area pathology on T2 MR imaging with motor disability in patients with acute flaccid myelitis.
\end{abstract}

MATERIALS AND METHODS: Spinal cord imaging for 9 patients with acute flaccid myelitis was analyzed by using the Spinal Cord Toolbox. A semiautomated pipeline using the Spinal Cord Toolbox measured lesion involvement in GM, WM, and total spinal cord cross-sectional area. Proportions of GM, WM, and cross-sectional area affected by T2 hyperintensity were calculated across 3 ROIs: 1) center axial section of lesion; 2) full lesion segment; and 3) full cord atlas volume. Spearman rank order correlation was calculated to compare MR metrics with clinical measures of disability.

RESULTS: Proportion of GM metrics at the center axial section significantly correlated with measures of motor impairment upon admission ( $r[9]=-0.78 ; P=.014)$ and at 3-month follow-up $(r[9]=-0.66 ; P=.05)$. Further, proportion of GM extracted across the full lesion segment significantly correlated with initial motor impairment $(r[9]=-0.74, P=.024)$. No significant correlation was found for proportion of WM or proportion of cross-sectional area with clinical disability.

CONCLUSIONS: Atlas-based measures of proportion of GM T2 signal abnormality measured on a single axial MR imaging section and across the full lesion segment correlate with motor impairment and outcome in patients with acute flaccid myelitis. This is the first atlas-based study to correlate clinical outcomes with segmented measures of $\mathrm{T} 2$ signal abnormality in the spinal cord.

ABBREVIATIONS: \%CSA = proportion of cross-sectional area; \%GM = proportion of gray matter; \%WM = proportion of white matter; AFM = acute flaccid myelitis; $\mathrm{EV}=$ enterovirus; $\mathrm{MRC}=$ Medical Research Council; SC = spinal cord; SCT = Spinal Cord Toolbox

A $\mathrm{fter}$ the eradication of wild poliovirus, the clinical syndrome of spinal motor neuron injury called acute flaccid myelitis (AFM) nearly disappeared from North America. The reemergence of this syndrome has coincided with the appearance of West Nile virus in Europe and North America, enterovirus (EV) A71 in

Received May 17, 2016; accepted after revision September 21.

From the Department of Radiology and Biomedical Imaging (D.B.M., J.F.T., M.D.M., Mark Wilson, J.N.), University of California, San Francisco, Zuckerberg San Francisco General Hospital and Trauma Center, San Francisco, California; Brain and Spinal Injury Center (J.F.T.), Zuckerberg San Francisco General Hospital and Trauma Center, San Francisco, California; Institute of Biomedical Engineering (J.C.-A.), Ecole Polytechnique Montreal, Montreal, Quebec, Canada; Functional Neuroimaging Unit (J.C.-A.), CRIUGM, University of Montreal, Montreal, Quebec, Canada; and Department of Neurology (Michael Wilson), University of California, San Francisco.

Please address correspondence to Jared A. Narvid, MD, University of California, San Francisco, Zuckerberg San Francisco General Hospital and Trauma Center, 1001 Potrero Ave, Room 1x57, SFGH Box 1325, San Francisco, CA 94110; e-mail: jared.narvid@ucsf.edu

- Indicates open access to non-subscribers at www.ajnr.org

http://dx.doi.org/10.3174/ajnr.A5044
Southeast Asia, and EV-D68 in North America. ${ }^{1,2}$ In fact, at the same time that EV-D68 caused widespread outbreaks of respiratory illness in the United States in 2014, the number of patients with AFM spiked. ${ }^{3}$ The Centers for Disease Control and Prevention noted this association, describing the incidence of AFM in a subset of patients with acute flaccid paralysis with evidence of spinal cord (SC) injury. ${ }^{4}$

More recently, longitudinally extensive SC lesions predominantly affecting the central GM have been described in children with AFM during the $2014 \mathrm{EV}$ outbreak. ${ }^{5}$ These patients reported acute limb weakness, cranial nerve dysfunction, or both. The prognosis in such patients is variable, with few predictors of recovery aside from the severity of initial paralysis. However, in a variety of SC pathologies, defining the pattern and extent of signal abnormality on MR imaging can aid in diagnosis and prognosis. ${ }^{6}$

The recent development of a standard SC template ${ }^{7}$ - as part of the Spinal Cord Toolbox (SCT) ${ }^{8}$ - that includes probabilistic maps of GM and $\mathrm{WM}^{9}$ now makes it possible to quantify the severity of SC 
injury. ${ }^{10-12}$ We aimed to measure the proportion of GM and WM damage in patients with AFM occurring in association with the EV-D68 outbreak. We hypothesized that the degree of GM and WM signal abnormality in these patients would both correlate with severity of initial symptoms and symptoms at follow-up after hospitalization. Although several studies have investigated the association between GM and WM signal abnormality on MR imaging and disease severity, ${ }^{13-18}$ to date, this is first study using this analysis method to register SCs with abnormal signal to an unbiased average anatomic template; quantify proportions of GM (\%GM), WM (\%WM), and CSA (\%CSA) occupied by lesion in the probabilistic template; and correlate data with clinical outcomes.

\section{MATERIALS AND METHODS \\ Case Definition}

All patients (9 total) admitted to the Benioff Children's Hospital at the University of Califronia, San Francisco with AFM were included in the study. Dates of hospital admission ranged from February 12, 2012, to February 2, 2015. All patients included in the study met the clinical case definition of AFM as described by the Centers for Disease Control and Prevention. Patients were defined as having acute limb weakness with MR imaging SC abnormality. Other infectious causes of AFM were excluded, such as Guillain-Barré syndrome, West Nile virus, poliovirus, stroke, transverse myelitis, myasthenia gravis, and botulism. Nasopharyngeal swab, oropharyngeal swab, serum, stool, or CSF samples were tested for EV RNA by using polymerase chain reaction for all patients. EV polymerase chain reaction was conducted at ViraCor for 3 patients, California Department of Public Health-Neurologic Testing and Surveillance Viral \& Rickettsial Disease Laboratory for 2 patients, Kaiser Permanente Medical Group for 2 patients, Lucile Packard Children's Hospital for 1 patient, and Stanford University Hospitals for 1 patient.

During initial hospitalization, all patients underwent complete neurologic testing. Strength testing was formalized with the composite Medical Research Council (MRC) Scale for Muscle Strength scores, using 3 proximal muscles and 2 distal muscles in the affected limb for a score ranging from $0-25 .{ }^{19}$ At follow-up, MRC strength testing was repeated and recorded. Improvement in strength was recorded as the numeric difference in MRC score.

Local institutional review board approval was obtained through the University of California, San Francisco Committee on Human Research for retrospective review and analysis of patient clinical information and MR images.

\section{MR Imaging Acquisition Parameters}

All MR imaging studies were performed on a $1.5 \mathrm{~T}$ Genesis HDxt Signa scanner with software version 15 (GE Healthcare, Milwaukee, Wisconsin). Axial and sagittal T2 FSE imaging was performed with the following parameters (presented as mean \pm SD from all 9 examinations): TR, $3398.78 \mathrm{~ms} \pm$ $1430.99 \mathrm{~ms}$; TE, $99.04 \mathrm{~ms} \pm 12.93 \mathrm{~ms}$; section thickness, 3.33 $\mathrm{mm}$; echo-train length, $18.56 \pm 3.28$. Average native plane resolution after interpolation of images is ( $2 \mathrm{D}$ to $3 \mathrm{D})$ : $\mathrm{X}, 0.40$; $\mathrm{Y}, 0.40 ; \mathrm{Z}, 4.17$. Additional sequences performed as part of our routine spine and brain MR imaging protocol were not evaluated for the purposes of this study.

\section{Image Processing}

T2-weighted images for 9 patients with AFM were analyzed with SCT. The FMRIB Software Library v5.0 (FSL; http://fsl.fmrib.ox.ac. $\mathrm{uk} /)^{20}$ viewer module was used to manually mark the seed points for analysis. These locations flag the beginning and end regions for the propagation deformation model to segment the SC. ${ }^{21}$ During automatic segmentation, detection of the SC is done in the axial plane by using the Hough transform. This is followed by propagation of an elliptical triangular tubular mesh built inside the SC. The tubular mesh is then deformed toward the edges of the SC. ${ }^{21}$ Results of segmentation accuracy were unsatisfactory because of the large signal hyperintensity in this cluster of patients. Because of this, segmentation of normal tissue was done using the SCT segmentation algorithm. Areas where segmentation deviated from the SC as a result of hyperintensity were manually adjusted by creating images of SC voxel locations by using FSL and 2 centerline points to mark the beginning and end axial sections for registration. Registration to the template took roughly $540 \mathrm{sec}-$ onds per patient. The SCT automatic pipeline to register, warp, and extract WM and GM metrics for each SC consisted of (Fig 1):

1) A $3 \mathrm{D}$ labels file was created based on the coordinates of the first (C2-C3) and last (through T6) vertebral level landmarks to be analyzed by using the SC labeling utility;

2) A 3D mask file was created manually in FSL to identify voxels within the SC. SC masks were confirmed by 2 radiologists as identifying only areas within the SC;

3) A combination of affine and nonlinear registrations was done of the T2-weighted image to the corresponding MNI-PolyAMU template. The MNI-Poly-AMU template is an image of the SC averaged across multiple patients and is used as a reference point for registration of patient SCs and atlas-based MR signal quantification. ${ }^{7}$ Registration was done in 4 steps. In step 1, a nonlinear transformation was estimated to straighten the SC (to match the MNI-Poly-AMU straight template). In step 2, an affine transformation was found based on the input labels to match the vertebral levels between the subject and the template. In step 3, a $2 \mathrm{D}$ section-wise registration was done to bring the subject closer to the template, while ensuring robustness toward pathology by using the cord segmentation instead of the image, by using the mean-square metric and smoothing factor of 2 . In step 4, local registration adjustment was made using the B-Spline SyN algorithm with the mean-square metric, gradient step of 0.5 , and smoothing factor of 0 , with 5 iterations. The outputs were a T2weighted image warped to the template and a template warped to the T2-weighted image, along with a pair of forward and reverse deformation fields;

4) The reverse deformation field (template to subject) was then applied to the WM and GM atlases, projecting them in the subject space. These warping fields were used to register multiparametric data to a common space for quantification of image-derived metrics;

5) Raw images were thresholded to provide binary ROI of lesion area for analysis with the WM/GM and SC probabilistic atlases. Thresholding of images was performed in ImageJ (National Institutes of Health, Bethesda, Maryland), ${ }^{22}$ with manual percentile adjustment to segment the lesions from surrounding normalappearing SC. Thresholded T2 images were confirmed by 2 neu- 


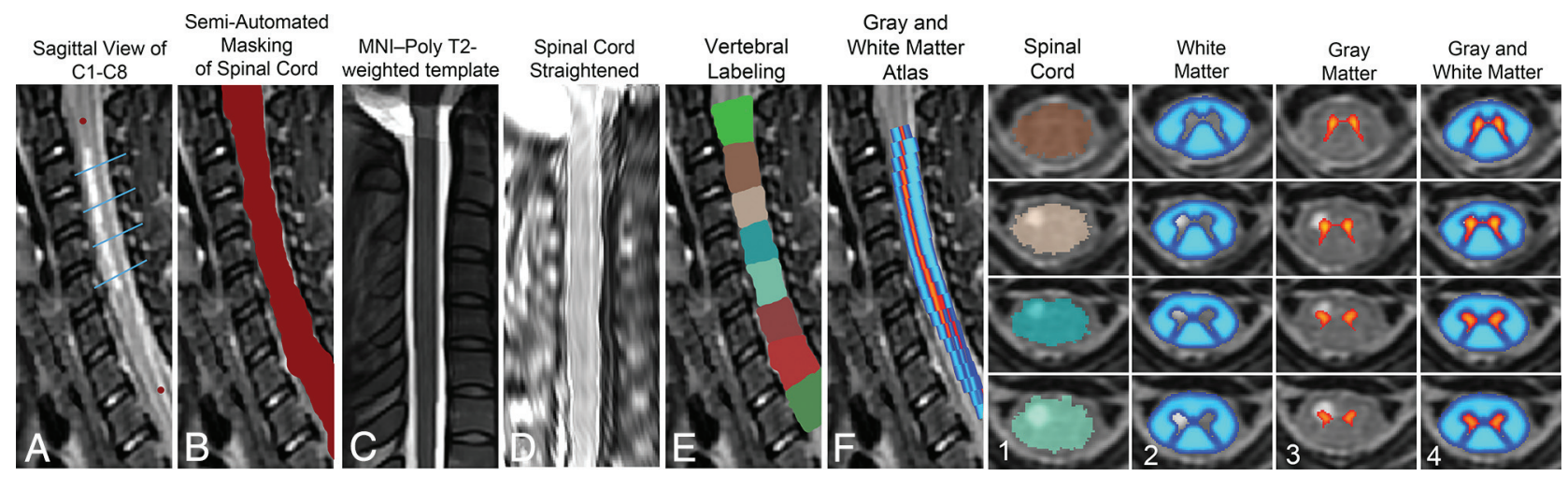

FIG 1. Steps for registering patient 5 (acute flaccid paralysis) to template. A, Red circles indicate manual marking of anatomic features $\mathrm{Cl}$ and $\mathrm{C} 8$. Blue lines indicate axial sections illustrated in far right grid. B, Manual masking of SC centerline was done at each axial section because of signal hyperintensity interfering with automatic reconstruction of centerline. $C, \mathrm{MNI}$-Poly-AMU T2-weighted template. D, SC straightening using thin-plate spline interpolation. E, Labeling of vertebral levels after registering to template and warping back to native space. $F$, Sagittal view of GM and WM probabilistic atlas after registering to template and warping back to native space. Far right grid, Output of template-based atlas. Column 1, Automatic vertebral body labeling and SC space. Column 2, Probabilistic masks of WM. Column 3, Probabilistic masks of GM. Column 4, Probabilistic masks of GM and WM overlaid.
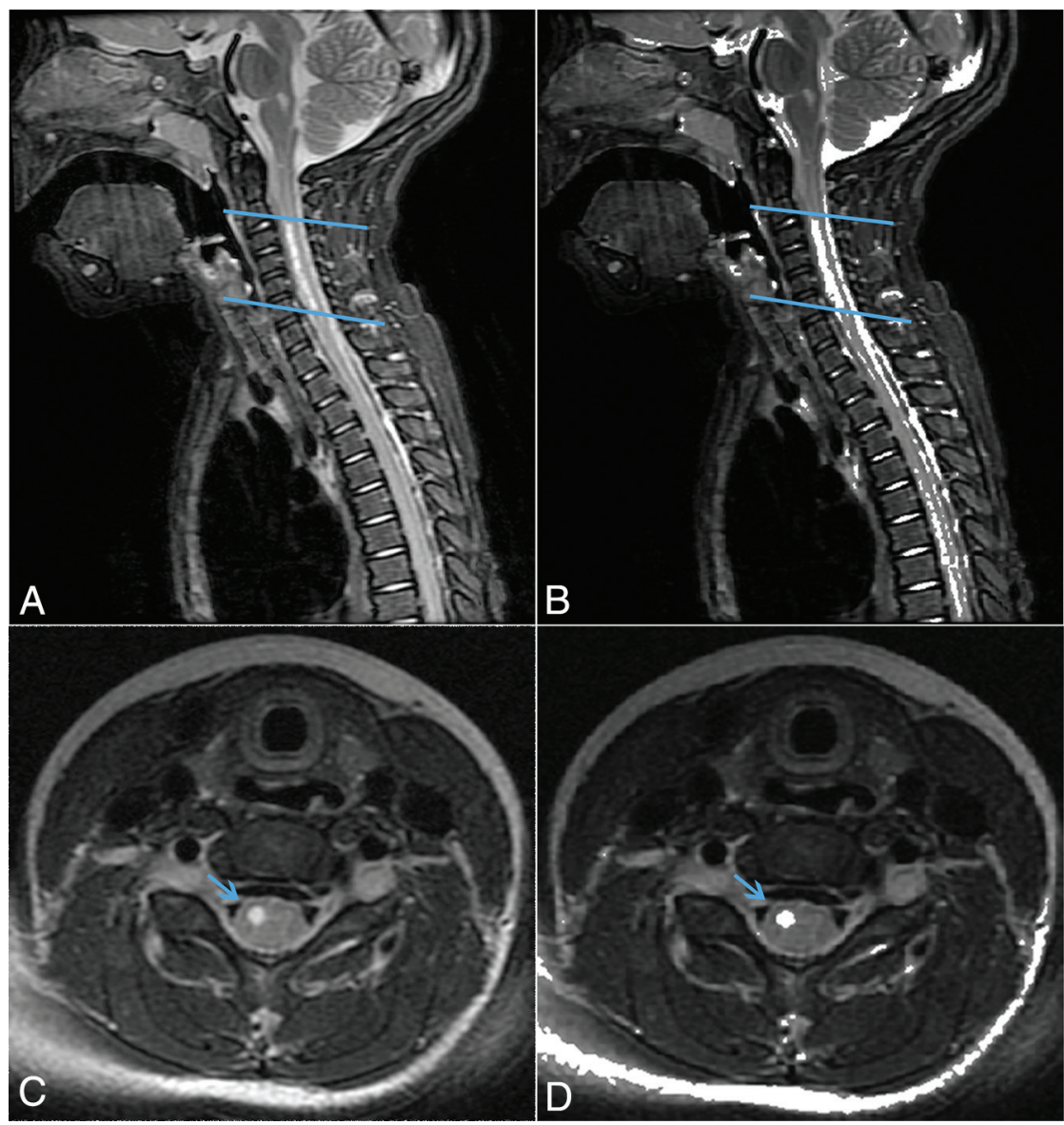

FIG 2. MR imaging of patient 1. A, Sagittal plane T2-weighted image centered on lesion. B, Overlay of binary thresholded image for lesion and T2-weighted sagittal image. C, Axial T2-weighted image at lesion center. D, Overlay of binary thresholded image for lesion and T2-weighted axial image.

roradiologists (J.N., J.F.T.) who were blinded to clinical data, segmenting only areas affected by lesions. Metrics were extracted by using the SCT extract metric function. The percentage of lesion within GM/WM and SC probabilistic atlas voxel space was extracted as a cumulative partial volume by using the thresholded MR images;
6) Cumulative partial volume metrics for GM and WM were extracted from the lesion axial center, lesion segment, and full cord atlas volume. A binary image of lesion area in this analysis is used to summate the voxel-wise probabilities occupied by the lesion per axial section;

7) Spearman rho $(r), P$ values, linear regression slopes, and intercepts were calculated by NumPy and matplotlib in Python. Regression plots and bar charts were also plotted using matplotlib; and,

8) To quantify differences in thresholding, binary images, which were thresholded by each neuroradiologist, were overlaid by using ImageJ. Pixels identifying areas of the images that were different between the reviewers were summed and divided by total pixels of the axial section image. These metrics are reported as the percentile average, $\mathrm{SD}$, and range of pixel differences.

\section{Atlas-Based Spinal Cord Injury Assessment}

Probabilistic maps containing all cervical levels and extending to T6 were created for all 9 patients. For analysis at the lesion center, 2 neuroradiologists (J.N., J.F.T.) reviewed each SC image and identified the axial section showing the most significant T2 hyperintensity, the so-called "lesion center." In 1 patient, the lesion center showing the most extensive hyperintensity could not be analyzed because it was below T6. In this case (patient 1), the second-most severely affected region was analyzed. For analysis of the full lesion segment, sagittal T2 images 


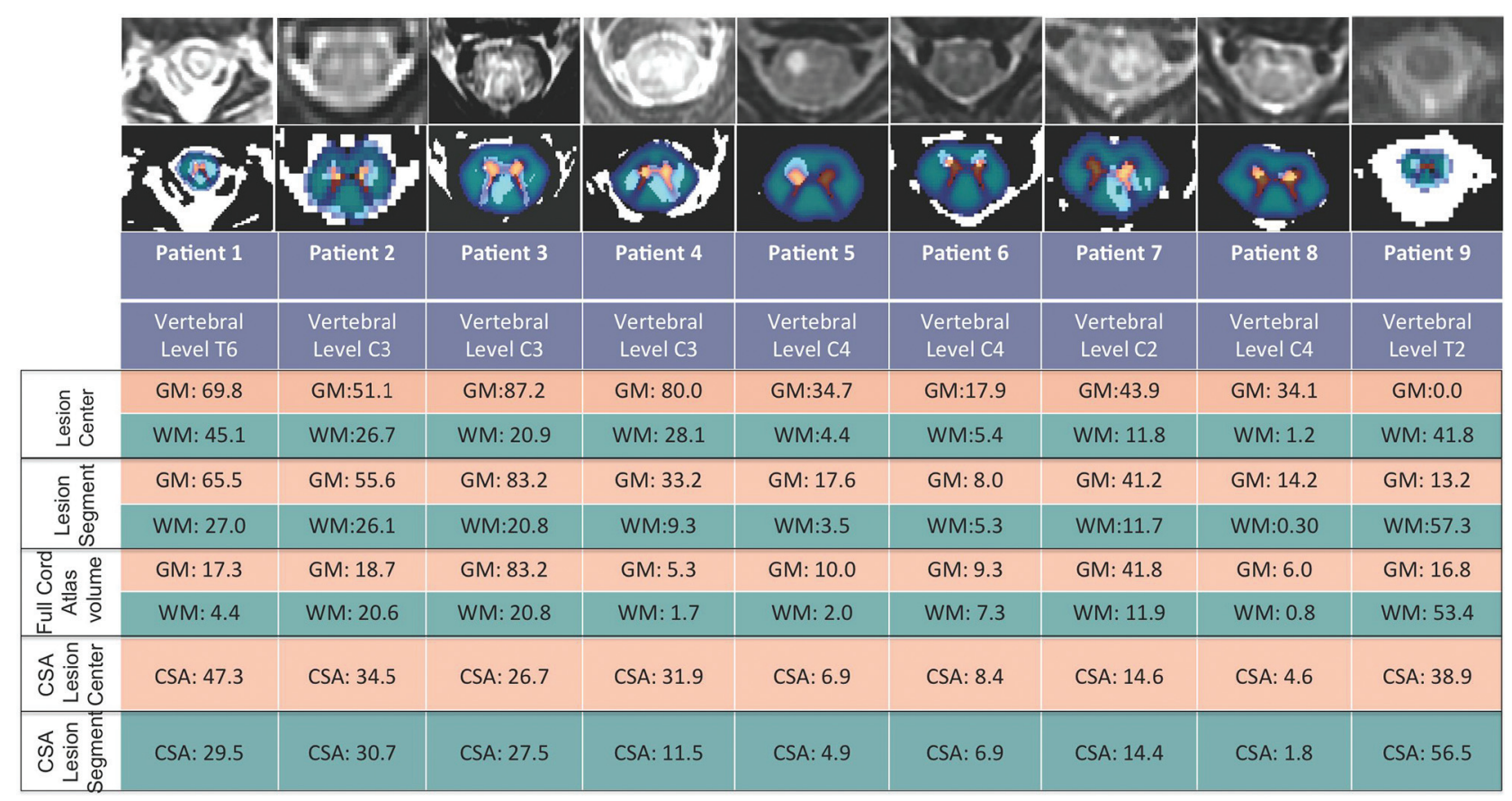

FIG 3. First row, Axial T2 image from center of lesion. Second row: Probabilistic GM (orange) and WM (blue) map overlaid on thresholded axial T2 image from lesion center. Lesion Center indicates \%WM and \%GM weighted average metrics at axial lesion center. Lesion Segment indicates \%WM and \%GM weighted average metrics for lesion segment. Full Cord Atlas Volume indicates \%WM and \%GM weighted average metrics for full cord atlas volume. CSA Lesion Center indicates \%CSA weighted average metrics at axial lesion center. CSA Lesion Segment indicates \%CSA weighted average metrics at lesion segment.

\begin{tabular}{|c|c|c|c|c|c|c|c|c|}
\hline $\begin{array}{l}\text { Patient } \\
\text { No. }\end{array}$ & $\begin{array}{c}\text { Age, } \\
\text { yr }\end{array}$ & Sex & $\begin{array}{c}\text { Limb } \\
\text { Weakness }\end{array}$ & $\begin{array}{c}\text { MRC } \\
\text { Composite } \\
\text { Score } \\
\end{array}$ & $\begin{array}{c}\text { Enterovirus } \\
\text { Detected }\end{array}$ & $\begin{array}{c}\text { MRC Score } \\
\text { at Follow-Up }\end{array}$ & Discharge Diagnosis & $\begin{array}{c}\text { Days to } \\
\text { MRI }\end{array}$ \\
\hline 1 & 3 & M & Right UE & 11 & NP swab & 2 & Disease due to $\mathrm{EV}$ & 9 \\
\hline 2 & 7 & M & Bilateral LE & 20 & None & 3 & Encephalomyelitis, paraesthesia/hyperestheia & 14 \\
\hline 3 & 4 & $\mathrm{~F}$ & Bilateral LE & 9 & None & 1 & Flaccid paralysis, unspecified & 2 \\
\hline 4 & 8 & M & Right UE & 9 & NP swab & 0 & Acute flaccid paralysis & 13 \\
\hline 5 & 27 & M & Bilateral LE & 1 & Serum & 3 & Virus-related myelitis & 6 \\
\hline 6 & 8 & $\mathrm{~F}$ & Left UE & 16 & None & 2 & Viral meningitis & 11 \\
\hline 7 & 24 & M & Bilateral LE & 24 & None & 3 & Meningomyelitis & 5 \\
\hline 8 & 10 & M & Left UE & 18 & None & 2 & Postinfectious mycoplasma transverse myelitis & 3 \\
\hline 9 & 2 & $\mathrm{~F}$ & Left UE & 3 & None & 1 & Hopkins syndrome & 1 \\
\hline
\end{tabular}

Note:-LE, lower extremities; NP, nasophyryngeal; UE, upper extremities.

were used to identify the starting and ending axial section where the lesion was involved (Fig 2). In the full cord atlas volume analysis, the entire length of thresholded SC within the probabilistic atlases was used. Cumulative partial volumes for GM, WM, and SC affected by lesion at the center axial section, full lesion segment, and full cord atlas volume for each patient are described in Fig 3. A Spearman rank order correlation was used to determine whether metrics from each lesion analysis significantly correlated with MRC strength scores or clinical outcome scores.

\section{Statistical Analysis}

Composite MRC for Muscle Strength scores, clinical outcomes scores, and cumulative partial volumes of the binary thresholded image in probabilistic GM and WM voxel space were used in a Spearman rank order correlation. $P$ values comparing patients testing as EV positive and EV negative were calculated by the Mann-Whitney $U$ test. Furthermore, Mann-Whitney $U$ tests for medians were used to determine whether GM and WM cumulative partial volumes were significantly different between outcome and MRC groups. An independent-samples Kruskal-Wallis test on GM metrics was used to compare groups. Spearman rank order correlations were also used to determine the correlation between the neuroradiologists' evaluation of the center axial section showing the most hyperintensity, the vertebral body with the most hyperintensity, and the thresholding level necessary to successfully segment the lesion from surrounding SC space. The Student $t$ test was used to determine whether the pixel percent differences from thresholding were significantly different from 0 . An $\alpha$ of .05 was considered statistically significant for all tests of association. No mathematic correction was made to adjust the $\alpha$ level for multiple corrections because the comparisons were planned before data inspection. 


\section{Lesion Center}
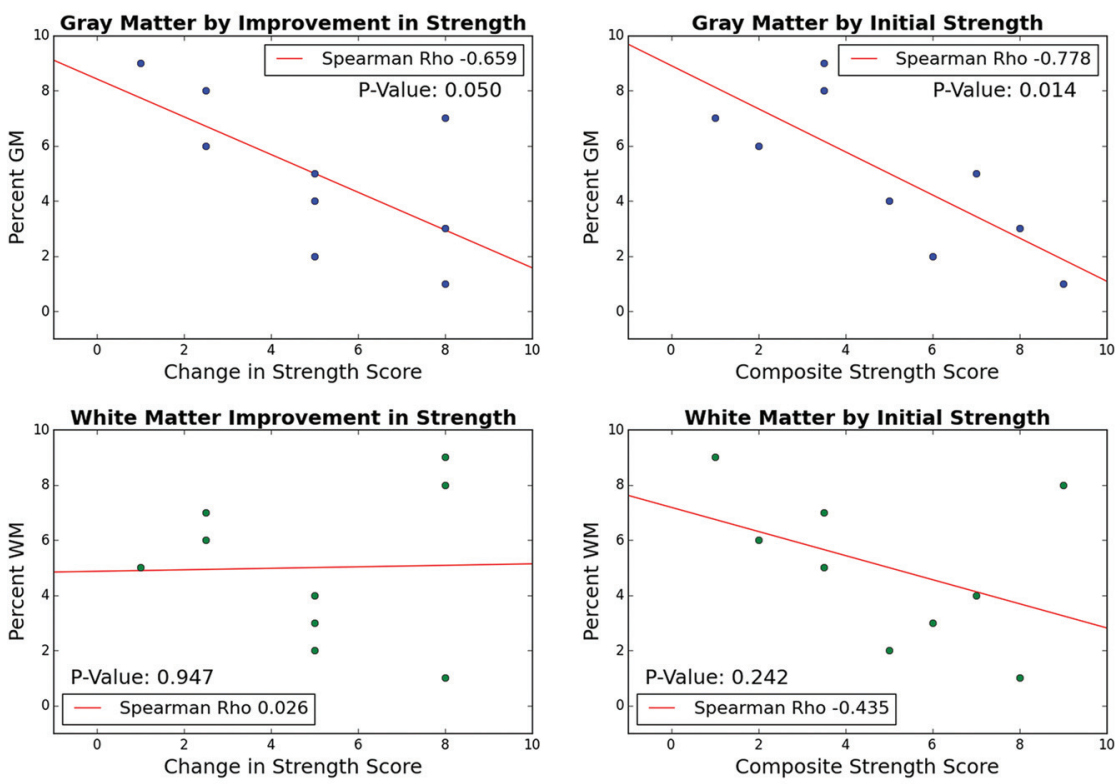

Lesion Segment
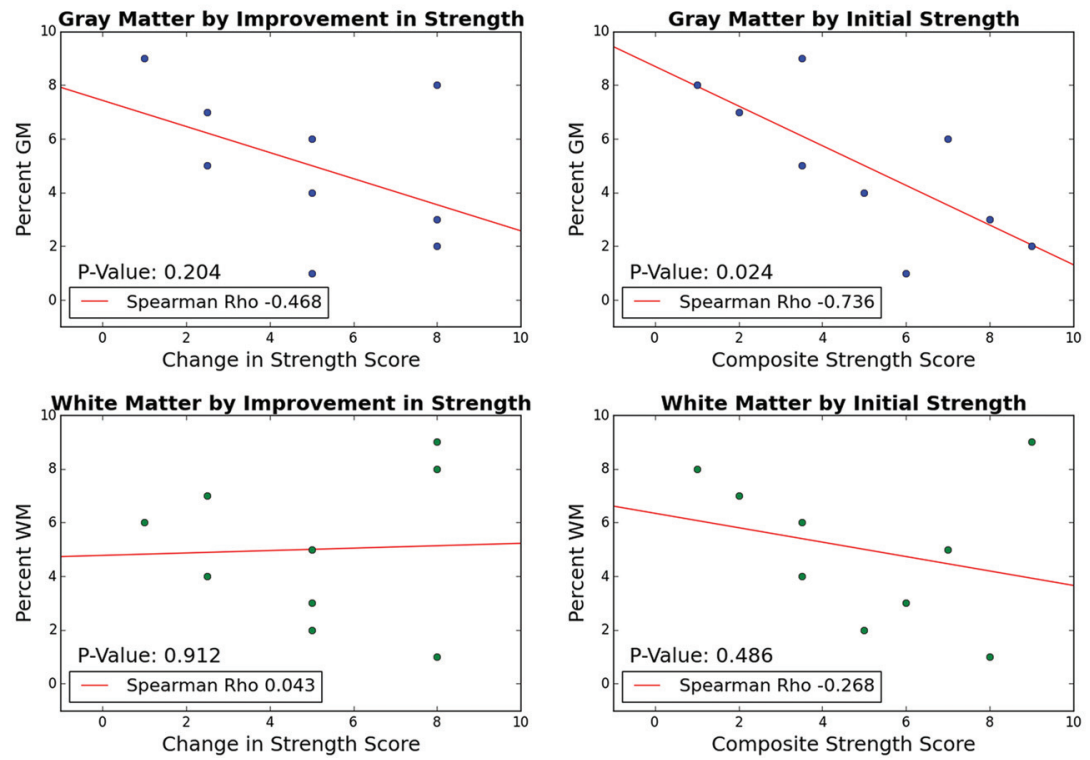

FIG 4. Scatterplots of numeric outcome of initial strength or improvement in strength MRC score by \%GM or \%WM injury (weighted average metric ranking) separated by analysis types (lesion center and lesion segment).

\section{RESULTS}

\section{Clinical Findings}

Patients were of age 2-27 years at the time of study, with most patients being younger than 10 years old ( 6 male, 3 female). The patient demographics, discharge diagnosis, MRC composite score, and clinical outcome rating are detailed in the Table. Additional presenting symptoms included fever (8 patients), upper respiratory infection (4 patients), body pain/pruritus/allodynia/abnormal sensation (5 patients), nausea/food intolerance/emesis (3 patients), urinary retention ( 2 patients), and ataxia (1 patient). EV polymerase chain reaction of the CSF was negative in all patients. Nasopharyngeal swabs tested positive for EV RNA in 2 patients and serum samples for 1 patient tested positive for EV. One nasopharyngeal swab sample was positively subtyped as EV-D68. Mean time between clinical symptoms and MR imaging was 6.89 days (interquartile range, 3-11 days) (Table). Mean clinical follow-up time was 396.33 days (interquartile range, 262-362 days).

\section{MR Imaging Findings}

All patients had SC lesions involving the central cord GM and some degree of surrounding WM. Lesions consisted of well-defined T2 hyperintensity predominantly within anterior horn cells for 6 patients $(2,3,4,5,6,8)$ and ill-defined lesions affecting the entire central SC GM for 3 patients $(1,7,9)$ (Fig 3). In 1 case, ill-defined T2 hyperintensity extended the entire length of the SC. Patients with the most longitudinally extensive hyperintensity throughout both cervical and thoracic areas had bilateral flaccid lower extremities. On MR T2 images, brain lesions were identified in 2 patients. One patient showed hyperintensity in the right frontal lobe. The second patient showed brain stem and thalamic edema.

\section{Spinal Cord Analysis}

MR SC injury metrics were calculated for each patient (Fig 3). Measuring at the center of the lesion (lesion center section), clinical outcome significantly worsened as \%GM increased ( $r$ [9] = $-0.66, P=.05$ ) (Fig 4). Similarly, the $\% \mathrm{GM}$ injured showed significant correlation with weakness at initial examination for both lesion center measurement $(r[9]=-.78, P=.014)$ and full lesion segment volume measurements $(r[9]=$ $-0.74, P=.024)$. There was no significant association between \%WM injured and clinical outcome or MRC strength scores at lesion center or full lesion segment volume. In full cord atlas volume analysis, neither GM nor WM significantly correlated with either improvement or initial weakness. No significant correlation was found between \%CSA at lesion center and clinical outcome $(r[9]=0.03, P=.95)$ or initial MRC score $(r[9]=-.46, P=.213)$. Furthermore, cumulative partial volumes extracted for \%CSA at lesion segment did not significantly correlate with clinical outcome $(r[9]=-0.05, P=$ $.89)$ or initial MRC score $(r[9]=-.25, P=.52)$. No significant differences were found between the EV-positive and EV-negative groups or among clinical outcome groups in the degree of initial weakness (strength score), extent of GM or WM injury in either lesion center, or full lesion segment volume. 
Selection of the axial section showing the most hyperintensity had good concordance between the 2 neuroradiologists (J.N., J.F.T. $)(r[9]=0.60, P=.088)$. Differences in axial section selection were mostly caused by several regions showing the same degree of hyperintensity. A strong degree of correlation was found between the neuroradiologists with regard to the vertebral body of the most hyperintensity $(r[9]=0.92, P<.001)$ and with regard to level of thresholding $(r[9]=0.98, P<.001)$. Average pixel differences as a result of thresholding variation between the neuroradiologists was $0.86 \%$ (SD, $1.87 \%$; range, $0 \%-5.8 \%$ ). A 2 -tailed $t$ test comparing the percentage difference to 0 showed no significant difference $(P=.19)$.

\section{DISCUSSION}

In the present study, we have used a semiautomated analysis pipeline to quantify T2 signal abnormality in the SC of patients diagnosed with AFM. More specifically, T2-weighted MR imaging sequences from 9 patients with AFM were successfully registered to the recently developed MNI-Poly-AMU SCT. Using SCT, measures of \%GM, \%WM, and \%CSA pathologic T2 signal hyperintensity were derived after thresholded T2 images segmented relative to pathologic signal were compared with probabilistic GM, WM, and SC maps from SCT. The primary aim of this study was to determine the feasibility and prognostic validity of implementing an atlas-based approach for MR imaging analysis in a population of patients with AFM. To this end, the positive correlations observed between SCT-derived MR imaging metrics of GM pathology and clinical outcome validate this approach. Although atlas-based analysis techniques have been applied to a variety of brain pathologies, ${ }^{23,24}$ this is the first study to implement an atlasbased approach for study of WM- and GM-specific pathology in the SC.

Patients with AFM occurring in association with a recent EVD68 outbreak were studied because this type of myelopathy distinctively targets central GM, primarily the anterior horn cells. ${ }^{25}$ Accordingly, an atlas that allows specific evaluation of GM signal abnormality would improve our ability to assess degree of injury and prognosis. In precisely this way, \%GM signal abnormality on a single axial section at the injury center most strongly correlated with neurologic impairment, whereas statistically insignificant correlations were seen with measures of \%WM and \%CSA injury. This is despite the presence of T2 signal abnormality involving WM to a varying degree in all patients. These findings are consistent with the presumed underlying pathophysiology of disease in this cohort of patients, wherein anterior horn cells of SC GM are particularly vulnerable to enteroviral toxicity and manifestations of their injury would be expected to best predict outcome. ${ }^{26}$ Other acute myelopathies, such as autoimmune demyelinating disease and traumatic contusion injury, often involve some component of direct myelin and axonal injury in WM with associated disruption of functionally significant ascending and descending WM tracts. ${ }^{23}$ The relative prognostic significance of GM pathology on MR imaging in this cohort of patients with AFM reflects the primary injury mechanism of AFM and highlights the value of segmented evaluation of the SC with distinct GM and WM maps.

The \%GM T2 signal hyperintensity on a single axial section at the most affected level of the injury center (referred to as "lesion center") provided the strongest correlation with clinical measures of motor impairment and recovery. The value of assessing the transverse extent of T2 abnormality on a single axial section at the injury center has been similarly demonstrated in the setting of acute traumatic SC injury and compressive myelopathy, ${ }^{18,27}$ suggesting that the transverse extent of injury in the SC on MR imaging carries significant diagnostic and prognostic information in a variety of pathologies. This result is significant because it allows for a more focused, rapid evaluation of the MR imaging findings centered at the injury center. The full lesion segment volume of \%GM signal abnormality also significantly correlated with initial motor scores and, to a lesser extent, with improvement. One potential advantage of full lesion segment volume calculation is that it does not rely upon the subjective determination of the most severely affected axial section, thus potentially reducing variability and making analysis more conducive to a fully automated process. When the \%GM involvement is calculated relative to the entire interrogated SC GM volume, the significance of this measure with motor scores is lost, likely as a result of the dilution of the pathologic signal within significantly larger volumes of normal GM when lesions are not longitudinally extensive.

Potential applications for SCT in quantifying SC pathology on MR imaging are vast and may greatly advance data-driven, unbiased approaches for assessing injury severity and guiding and monitoring therapy. $7,9,11,21,23,28$ This proof-of-concept study demonstrates the prognostic validity of this approach and how segmented analysis of SC subregions (ie, \%GM and \%WM) may reflect the underlying pathophysiology of disease.

\section{Limitations}

The intramedullary $\mathrm{T} 2$ hyperintensity observed in our patients often resulted in obscuration of margins between SC and hyperintense CSF, thereby precluding completely automated SC segmentation and necessitating manual adjustment for this step. An algorithm that can robustly and accurately segment the SC in patient populations with intramedullary T2 hyperintensity would reduce time and any bias associated with manual segmentation of the SC. Furthermore, 3T imaging would improve this delineation, thereby improving automated segmentation. In the current study, the segmentation and thresholding process were both reviewed by fellowship-trained neuroradiologists; however, in order for large throughput analysis of patient SCs with minimal manual image postprocessing, the propagated segmentation algorithm will need the capacity to propagate through axial sections with abnormal signal intensity. In addition, nonautomated thresholding introduces the possibility of error and/or bias during the image processing. Another limitation in the current study is that signal abnormality below T6 cannot be analyzed because the MNI-Poly-AMU template only covers C1-T6 vertebral levels. This limitation resulted in 1 patient's most severe axial section being excluded from analysis and the second-most severe section being used. This limitation will be overcome with the future release of the new PAM50 template, which includes the brain stem and full SC. ${ }^{29}$ In addition, the existing template was created from healthy young control patients similar in age to our older patients, but did not include young children. Finally, power analysis for this study by using an $\alpha$ of .05, and 9 patients indicates that an $r$ 
value of 0.75 is required to detect a significant difference with a power of $80 \%$. Because of this, the current study is underpowered in determining significance for weaker correlations between atlas-derived quantitative metrics of tissue damage and clinical outcomes.

\section{CONCLUSIONS}

This proof-of-concept study used a recently developed opensource Spinal Cord Toolbox for atlas-based analysis of T2 signal abnormality in the SCs of 9 patients with AFM occurring during the EV-D68 outbreak in the western United States. This cluster of patients showed distinctive SC lesions of the anterior central GM, characteristic of EV myelopathy, with variable WM involvement. An image processing and analysis pipeline was developed to register thresholded T2 MR images to the MNI-Poly-AMU template, enabling calculation of \%WM, \%GM, and \%CSA pathology. Quantitative measures of \%GM signal abnormality on a single axial section at the most severely affected level of the injury epicenter were significantly associated with clinical outcome scores and MRC muscle strength scores, reflecting the underlying pathophysiology for these patients with AFM. In addition, cumulative partial volumes of \%GM involved in whole lesion segment were significantly correlated with MRC Muscle Strength scores. \%GMsegmented calculations outperformed \%WM and \%CSA for predicting motor outcome. To date, this is the first study to use an atlas-based approach to quantify T2 measures of pathology in the SC and correlate extracted metrics with clinical outcomes.

Disclosures: Jason Talbott-UNRELATED: Consultancy: StemCells, Inc, Comments: member of data monitoring committee for clinical trial. Michael Wilson-UNRELATED: Grant: National Center for Advancing Translational Sciences of the National Institutes of Health, Comments: award number KL2TR000143. Julien Cohen-AdadUNRELATED: Consultancy: Sanofi, Biospective, Massachusetts General Hospital; Employment: Ecole Polytechnique Montreal, Functional Neuroimaging Unit (University of Montreal); Grants/Grants Pending: Canadian Institutes of Health Research, Natural Sciences and Engineering Research Council, Fonds de Recherche du Québec Nature et Technologies (FQRNT), Fonds de Recherche du Québec - Santé (FRQS), Canada Research Chair*; Royalties: Elsevier. *Money paid to the institution.

\section{REFERENCES}

1. Pons-Salort M, Parker EP, Grassly NC. The epidemiology of nonpolio enteroviruses: recent advances and outstanding questions. Curr Opin Infect Dis 2015;28:479-87 CrossRef Medline

2. Greninger AL, Naccache SN, Messacar K, et al. A novel outbreak enterovirus D68 strain associated with acute flaccid myelitis cases in the USA (2012-14): a retrospective cohort study. Lancet Infect Dis 2015;15:671-82 CrossRef Medline

3. Messacar K, Schreiner TL, Maloney JA, et al. A cluster of acute flaccid paralysis and cranial nerve dysfunction temporally associated with an outbreak of enterovirus D68 in children in Colorado, USA. Lancet 2015;385:1662-71 CrossRef Medline

4. Division of Viral Diseases, National Centers for Immunization and Respiratory Diseases, CDC; Division of Vector-Borne Diseases, Division of High-Consequence Pathogens and Pathology, National Center for Emerging and Zoonotic Infectious Diseases, CDC; Children's Hospital Colorado; Council of State and Territorial Epidemiologists. Notes from the field: acute flaccid myelitis among persons aged $\leq 21$ years-United States, August 1-November 13, 2014. MMWR Morb Mortal Wkly Rep 2015:63:1243-44 Medline

5. Maloney JA, Mirsky DM, Messacar K, et al. MRI findings in children with acute flaccid paralysis and cranial nerve dysfunction occurring during the 2014 enterovirus D68 outbreak. AJNR Am J Neuroradiol 2015;36:245-50 CrossRef Medline
6. Baruah D, Chandra T, Bajaj M, et al. A simplified algorithm for diagnosis of spinal cord lesions. Curr Probl Diagn Radiol 2015;44: 256-66 CrossRef Medline

7. Fonov VS, Le Troter A, Taso M, et al. Framework for integrated MRI average of the spinal cord white and gray matter: the MNIPoly-AMU template. Neuroimage 2014;102(Pt 2):817-27 CrossRef Medline

8. Cohen-Adad J, De Leener B, Benhamou M, et al. Spinal Cord Toolbox: an open-source framework for processing spinal cord MRI data. In: Proceedings of the 20th Annual Meeting of Organization for Human Brain Mapping, Hamburg, Germany. June 8-14, 2014

9. Lévy S, Benhamou M, Naaman C, et al. White matter atlas of the human spinal cord with estimation of partial volume effect. Neuroimage 2015;119:262-71 CrossRef Medline

10. Ullmann E, Pelletier Paquette JF, Thong WE, et al. Automatic labeling of vertebral levels using a robust template-based approach. Int J Biomed Imaging 2014;2014:719520 CrossRef Medline

11. Yiannakas MC, Mustafa AM, De Leener B, et al. Fully automated segmentation of the cervical cord from T1-weighted MRI using PropSeg: application to multiple sclerosis. Neuroimage Clin 2016; 10:71-77 CrossRef Medline

12. Martin AR, De Leener B, Aleksanderek I, et al. A prospective longitudinal study in degenerative cervical myelopathy using quantitative microstructural MRI with tract-specific metrics. In: Proceedings of the 24th Annual Meeting of International Society for Magnetic Resonance in Medicine, Suntec City, Singapore. May 7-13, 2016

13. Okuda DT, Mowry EM, Cree BA, et al. Asymptomatic spinal cord lesions predict disease progression in radiologically isolated syndrome. Neurology 2011;76:686-92 CrossRef Medline

14. Schlaeger R, Papinutto N, Zhu AH, et al. Association between thoracic spinal cord gray matter atrophy and disability in multiple sclerosis. JAMA Neurol 2015;72:897-904 CrossRef Medline

15. Kearney H, Miller DH, Ciccarelli O. Spinal cord MRI in multiple sclerosis-diagnostic, prognostic and clinical value. Nat Rev Neurol 2015;11:327-38 CrossRef Medline

16. Bede P, Bokde AL, Byrne S, et al. Spinal cord markers in ALS: diagnostic and biomarker considerations. Amyotroph Lateral Scler 2012; 13:407-15 CrossRef Medline

17. Mabray MC, Talbott JF, Whetstone WD, et al. Multidimensional analysis of magnetic resonance imaging predicts early impairment in thoracic and thoracolumbar spinal cord injury. J Neurotrauma 2016;33:954-62 CrossRef Medline

18. Talbott JF, Whetstone WD, Readdy WJ, et al. The Brain and Spinal Injury Center score: a novel, simple, and reproducible method for assessing the severity of acute cervical spinal cord injury with axial T2-weighted MRI findings. J Neurosurg Spine 2015;23:495-504 CrossRef Medline

19. Dyck PJ, Boes CJ, Mulder D, et al. History of standard scoring, notation, and summation of neuromuscular signs. A current survey and recommendation. J Peripher Nerv Syst 2005;10:158-73 CrossRef Medline

20. Jenkinson M, Beckmann CF, Behrens TE, et al. FSL. Neuroimage 2012;62:782-90 CrossRef Medline

21. De Leener B, Kadoury S, Cohen-Adad J. Robust, accurate and fast automatic segmentation of the spinal cord. Neuroimage 2014;98: 528-36 CrossRef Medline

22. Schneider CA, Rasband WS, Eliceiri KW. NIH Image to ImageJ: 25 years of image analysis. Nat Methods 2012;9:671-75 CrossRef Medline

23. Martin AR, Aleksanderek I, Cohen-Adad J, et al. Translating stateof-the-art spinal cord MRI techniques to clinical use: a systematic review of clinical studies utilizing DTI, MT, MWF, MRS, and fMRI. Neuroimage Clin 2016;10:192-238 CrossRef Medline

24. Evans AC, Janke AL, Collins DL, et al. Brain templates and atlases. Neuroimage 2012;62:911-22 CrossRef Medline

25. Tihan T. Pathologic approach to spinal cord infections. Neuroimaging Clin N Am 2015;25:163-72 CrossRef Medline 
26. Jubelt B, Lipton HL. Enterovirus/picornavirus infections. Handb Clin Neurol 2014;123:379-416 CrossRef Medline

27. You JY, Lee JW, Lee E, et al. MR classification system based on axial images for cervical compressive myelopathy. Radiology 2015;276: 553-61 CrossRef Medline

28. Hendrix P, Griessenauer CJ, Cohen-Adad J, et al. Spinal diffusion tensor imaging: a comprehensive review with emphasis on spinal cord anatomy and clinical applications. Clinical Anat 2015;28:88-95 CrossRef Medline 29. De Leener B, Taso M, Fonov V, et al. Fully-integrated T1, T2, T2*, white and gray matter atlases of the spinal cord. In: Proceedings of the 24th Annual Meeting of International Society for Magnetic Resonance in Medicine, Suntec City, Singapore. May 7-13, 2016 